\title{
The moving target of psychiatric diagnosis
}

\author{
Martin Alda, MD
}

Clinical diagnosis and/or phenomenological description is the cornerstone of both clinical care and research in psychiatry. Clinical management and guidelines are structured around individual diagnoses (e.g., major depression, schizophrenia, bipolar disorder), with the implicit understanding that such diagnoses mean the same to most clinicians (reliability) and that they have predictive value (validity). Another relevant aspect of diagnosis is its clinical utility - a measure that is difficult to estimate correctly. However, indirect data, such as increasing burden of psychiatric disorders, might be in part explained by diagnostic limitations. ${ }^{1}$

Similar considerations apply to research. While molecular, genetic, and brain imaging technologies have been making considerable progress, the phenotype definition frequently becomes a bottleneck with multiple ramifications: inconsistencies and difficulties in properly defining the phenotype lead to seemingly contradictory findings. It is my hope, however, that a careful examination of such discrepancies can be instructive and help move psychiatric research forward.

Let us consider some examples. An analysis of symptoms and life trajectories led to a proposal for a general psychopathology " $\mathrm{p}$ factor." 2 This $\mathrm{p}$ factor has been viewed as a single dimension underpinning the various psychiatric symptoms and explaining a large portion of interindividual variability with respect to presence of psychopathology. In parallel, results of genome-wide association studies led to a proposal of a common genomic $\mathrm{p}$ factor for psychiatric disorders. ${ }^{3}$ Similarly, the concept of general neurotic syndrome, while absent from current classification schemes, has existed for some time and is supported, for example, by twin data. ${ }^{4}$ Contrary to these concepts, in a family-based study of a Swedish population, Kendler and colleagues were able to separate rather clearly the 3 major psychiatric disorders (depression, bipolar disorder and schizophrenia). ${ }^{5}$ From family history data in health population registries, they constructed family genetic risk scores and compared them across the diagnoses. Each of the 3 conditions had minimal overlap except for elevated family genetic risk scores for depression in those with bipolar disorder, which is unsurprising given that finding's consistency with the results of older family studies. In an extended analysis that included a number of other diagnoses, the separation became less distinct, showing correlations between, for example, major depression and anxiety or substance use and attention-deficit/ hyperactvity disorder (ADHD). ${ }^{6}$

As of now, some of the largest genetic studies in psychiatry, counting tens or even hundreds of thousands of cases and hundreds of thousands controls, have identified at least 270 susceptibility loci for schizophrenia (preprint evidence: https: / /www.medrxiv.org/content/10.1101/2020.09.12.2019 2922v1), 64 for bipolar disorder, ${ }^{7} 102$ for major depression, ${ }^{8}$ as well as multiple loci for other diagnoses. Many of the genetic variants implicated in one condition seem to be involved in other diagnoses, leading to high genetic correlations between individual psychiatric diagnoses and suggesting shared heritability. ${ }^{9}$ Several recent genome-wide association studies found a substantial correlation between the genetic liabilities for most main psychiatric diagnoses (schizophrenia, bipolar disorder, major depressive disorder, autism, ADHD, or anxiety disorders). These results were obtained using mostly criteria-based diagnoses without any further refinement. Several investigations went even further in simplifying their recruitment and relied on limited phenotyping and/or self-reported diagnoses. Studies of major depression or bipolar disorder in self-identified individuals in databases such as 23andMe or the UK Biobank resulted in considerably weaker genetic associations and low so-called single nucleotide polymorphism (SNP) heritability (proportion of phenotypic variance explained by markers identified by genome-wide association analysis). ${ }^{7,10}$ Conversely, a recent study of the genetics of patients with major depressive disorder treated with electroconvulsive therapy (ECT) - arguably a much more severe and more specific diagnosis - yielded SNP heritability about 4 times greater than a standard genome-wide association study of major depression. ${ }^{11}$

In addition to the core psychiatric diagnosis, other features come into play. For instance, while structural brain imaging studies of bipolar disorder found reproducible differences from healthy unaffected individuals, it appears that some of the findings are not due to the disorder itself, but rather are secondary to metabolic factors such as obesity or insulin resistance that are frequently comorbid with severe mental illness. ${ }^{12}$

Correspondence to: M. Alda, Department of Psychiatry, Dalhousie University, Abbie J. Lane Building, room 3088, 5909 Veterans Memorial Lane, Halifax, NS B3H 2E2; malda @ dal.ca

DOI: $10.1503 / j p n .210098$ 
Research and psychiatric genetics in particular promised to uncover the true nature of psychiatric diagnoses and transform their classification. It may be the time to pause and ask whether this is happening and, if not, why. For a long time, psychiatry has existed with multiple diagnostic systems, operationalized criteria and tools to describe psychopathology. What strikes a casual observer is the amount of discontinuity among them.

First, DSM and ICD criteria are not the only classification systems. Numerous nosological concepts, classifications, psychopathology description systems, and definitions of individual disorders have been proposed. They differ in their history and purpose. What sets the current criteria apart is that they represent more or less broad consensus, whereas other systems are mostly based on theories of their time. The older classifications and/or disorder definitions were often developed by individual scholars and were frequently idiosyncratic, but they were also based on observations of large numbers of patients over extended periods of time and usually were not coloured by psychopharmacological treatments. The modern criteria, on the other hand, have been created by task forces and committees; they are usually descriptive, cross-sectional, reliable, atheoretical and - some argue — "sterile."13

Thus, we find a low degree of continuity among the different diagnostic and psychopathology systems that make it difficult to learn from older findings. The discontinuity is reflected in many areas, including epidemiological data or varying effectiveness of treatment interventions. The reasons for the described inconsistencies are many and include some of the following factors.

The meaning of some diagnoses changes over time. For instance, contrary to some claims, the DSM category of bipolar disorder differs from manic depressive insanity, as described by Kraepelin. ${ }^{14}$ Kraepelin's concept was both broader owing to its inclusion of recurrent depression and narrower because it stressed an episodic clinical course. Schizoaffective disorder was first proposed by Kasanin in 1933 as a specific form of recurrent psychotic state with affective symptoms, rapid onset and often clouded consciousness, ${ }^{15}$ while in modern criteria it is conceptualized as a condition meeting criteria for major mood disorder and for schizophrenia at different times. The broadening and narrowing of diagnostic categories have changed the definitions of several disorders, most dramatically autism (autism spectrum) and to a lesser extent ADHD (minimal brain dysfunction).

Some diagnoses that were arguably useful in the past have now been removed from the classification systems (e.g., simple schizophrenia, cycloid psychosis), and new ones have been added (e.g., schizotypal personality disorder).

The stigma of psychiatric diagnosis needs to be considered as well. The diagnoses of hysteria or histrionic personality were replaced first with that of borderline personality disorder and then euphemistically with "cluster B" personality/ traits, both of which had different meanings when initially defined than they do now. ${ }^{16}$

The clinical presentation is modified by external elements that change with time. Substance abuse is one such factor, with notable shifts both in terms of prevalence and the type and potency of drugs used. Iatrogenic factors are another poorly studied aspect (compare antidepressant-induced mixed states, rapid cycling, or super-sensitivity psychosis in long-term neuroleptic use).

As remarked by Cooper, "disorders are different from diseases." ${ }^{17}$ In psychiatry, perhaps few conditions could be considered diseases and/or meet the validity criteria, as postulated by Robins and Guze. ${ }^{18}$

Terminological shifts can be illustrated, for instance, in the concept of affective psychosis compared with mood disorders, seemingly referring to the same group of conditions. Affective psychosis did not automatically imply presence of hallucinations or delusions. In some earlier concepts it denoted mood that was grossly out of keeping with an individual's circumstances and/or that could not be explained by them. ${ }^{19}$ On the other hand, in DSM, mood disorders as such are not defined as affective psychosis; only individual mood episodes can have the specifier "with psychotic features," for which the presence of hallucinations and/or delusions is the necessary criterion.

Most psychiatrists accept that mental disorders are heterogeneous. But the lines of separation may not necessarily lie along subgroups defined by the current criteria. For example, there is an ongoing debate about the position of bipolar II disorder among mood disorders. ${ }^{20,21}$ It is conceivable that some cases of bipolar II disorder are milder variants of bipolar I disorder and that others are an entirely different condition (the same may apply to unipolar depression, with a minority of cases genetically related to bipolar I disorder). An example of a diagnosis not codified by any current criteria is that of lithium-responsive bipolar disorder. It includes both bipolar I and bipolar II diagnoses, and it is familial, longitudinally stable and appears to be associated with specific pathophysiological findings. ${ }^{22-24}$

An important limitation in current nosology is the loss of longitudinal perspective. Most psychiatric symptoms are nonspecific, and practically none are pathognomonic. Without appreciation for the evolution of psychopathology and the sequence and temporary (in)congruence of individual symptoms, clinicians would be missing a substantial part of the clinical picture. Psychiatric diagnosis is commonly based on the criterion of "change of function," or the observation that the symptoms are "out of character" for the patient. Yet, it is uncommon for psychiatrists in academic centres to follow patients longitudinally and see them when they are well. In a consultation model of specialist care, the link with patients' families is also less strong. As a result, some of the important clinical observations come from motivated individuals, often operating in private practice settings (compare the numerous contributions of Leonardo Tondo ${ }^{25}$ or Franco Benazzi ${ }^{26}$ in the field of mood disorders).

Better understanding of psychiatric disorders may emerge from a stepwise iterative approach, in which study of pathophysiological mechanisms will be based on careful clinical descriptions, and the findings will then inform the phenotypic delineations and either help separate 
more homogeneous subgroups or, conversely, combine previously distinct syndromes. Such approaches will need to rely not only on a cross-sectional description of a handful of symptoms, but will also have to consider the longitudinal course of these and control for important variables such as family history.

Affiliations: From the Department of Psychiatry, Dalhousie University, Halifax, NS, Canada.

Competing interests: None declared.

Content licence: This is an Open Access article distributed in accordance with the terms of the Creative Commons Attribution (CC BY-NC-ND 4.0) licence, which permits use, distribution and reproduction in any medium, provided that the original publication is properly cited, the use is noncommercial (i.e., research or educational use), and no modifications or adaptations are made. See: https:/ / creativecommons.org/licenses/by-nc-nd/4.0/

\section{References}

1. GBD 2019 Diseases and Injuries Collaborators. Global burden of 369 diseases and injuries in 204 countries and territories, 19902019: a systematic analysis for the Global Burden of Disease Study 2019. Lancet 2020;396:1204-22.

2. Caspi A, Houts RM, Belsky DW, et al. The p factor: One general psychopathology factor in the structure of psychiatric disorders? Clin Psychol Sci 2014;2:119-37.

3. Sprooten E, Franke B, Greven CU. The p-factor and its genomic and neural equivalents: an integrated perspective. Mol Psychiatry 2021;doi: 10.1038/s41380-021-01031-2

4. Andrews G, Stewart G, Morris-Yates A, et al. Evidence for a general neurotic syndrome. Br J Psychiatry 1990;157:6-12.

5. Kendler KS, Ohlsson H, Sundquist J, et al. Family genetic risk scores and the genetic architecture of major affective and psychotic disorders in a Swedish national sample. JAMA Psychiatry 2021;doi: 10.1001/jamapsychiatry.2021.0336 [online ahead of print].

6. Kendler KS, Ohlsson H, Sundquist J, et al. The patterns of family genetic risk scores for eleven major psychiatric and substance use disorders in a Swedish national sample. Transl Psychiatry 2021;11:326.

7. Mullins N, Forstner AJ, O'Connell KS, et al. Genome-wide association study of more than 40,000 bipolar disorder cases provides new insights into the underlying biology. Nat Genet 2021;53:817-29.

8. Howard DM, Adams MJ, Clarke TK, et al. Genome-wide metaanalysis of depression identifies 102 independent variants and highlights the importance of the prefrontal brain regions. Nat Neurosci 2019;22:343-52.

9. Bipolar Disorder and Schizophrenia Working Group of the Psychiatric Genomics Consortium. Genomic dissection of bipolar disorder and schizophrenia, including 28 subphenotypes. Cell 2018;173: 1705-1715.e16.
10. Cai N, Revez JA, Adams MJ, et al. Minimal phenotyping yields genome-wide association signals of low specificity for major depression. Nat Genet 2020;52:437-47.

11. Clements CC, Karlsson R, Lu Y, et al. Genome-wide association study of patients with a severe major depressive episode treated with electroconvulsive therapy. Mol Psychiatry 2021; doi: 10.1038/ s41380-020-00984-0 [online ahead of print].

12. McWhinney SR, Abe C, Alda M, et al. Association between body mass index and subcortical brain volumes in bipolar disorders ENIGMA study in 2735 individuals. Mol Psychiatry 2021;doi: 10.1038/s41380-021-01098-x [online ahead of print].

13. Carroll BJ. Neurobiologic dimensions of depression and mania. In: Angst J, editor. The origins of depression: Current concepts and approaches. Berlin: Springer-Verlag; 1983. p.163-86.

14. Kraepelin E. Manic-depressive insanity and paranoia (English translation by RM Barclay). E. \& S. Livingstone, Edinburgh, 1921.

15. Kasanin J. The acute schizoaffective psychoses. Am J Psychiatry 1994;151:144-54.

16. Hudziak JJ, Boffeli TJ, Kreisman JJ, et al. Clinical study of the relation of borderline personality disorder to Briquet's syndrome (hysteria), somatization disorder, antisocial personality disorder, and substance abuse disorders. Am J Psychiatry 1996;153:1598-606.

17. Cooper J. Disorders are different from diseases. World Psychiatry 2004;3:24.

18. Robins E, Guze SB. Establishment of diagnostic validity in psychiatric illness: its application to schizophrenia. Am J Psychiatry 1970;126:983-7.

19. Ban TA. Development of the diagnostic concept of manic-depressive psychosis in Emil Kraepelin's classifications. Córdoba: International Network for History of Neuropsychopharmacology; 2015 Availalble: https://inhn.org/no_cache/archives/ban-collection/development -of-the-diagnostic-concept-of-manic-depressive-psychosis-in-emil -kraepelins-classification.html (accessed 2021 June 12).

20. Parker G. Polarised views about bipolar disorder(s): a critique of the 2020 College guidelines for mood disorders. Aust N Z J Psychiatry 2021;55:548-52.

21. Malhi GS. Thing one and thing two(1): What 'doctors use' to doctor you? Aust N Z J Psychiatry 2021;55:536-47.

22. Alda M, Grof P, Rouleau GA, et al. Investigating responders to lithium prophylaxis as a strategy for mapping susceptibility genes for bipolar disorder. Prog Neuropsychopharmacol Biol Psychiatry 2005;29:1038-45.

23. Tobe BTD, Crain AM, Winquist AM, et al. Probing the lithiumresponse pathway in hiPSCs implicates the phosphoregulatory set-point for a cytoskeletal modulator in bipolar pathogenesis. Proc Natl Acad Sci U S A 2017;114:E4462-71.

24. Stern S, Santos R, Marchetto M, et al. Neurons derived from patients with bipolar disorder divide into intrinsically different subpopulations of neurons, predicting the patients' responsiveness to lithium. Mol Psychiatry 2018;23:1453-65.

25. Tondo L, Lepri B, Baldessarini RJ. Suicidal risks among 2826 Sardinian major affective disorder patients. Acta Psychiatr Scand 2007;116:419-28.

26. Benazzi F. What is hypomania? Tetrachoric factor analysis and kernel estimation of DSM-IV hypomanic symptoms. J Clin Psychiatry 2009;70:1514-21. 